\title{
Increased platelet activation in patients with stable and acute exacerbation of COPD
}

\author{
John D Maclay, ${ }^{1}$ David A McAllister, ${ }^{1,2}$ Shonna Johnston, ${ }^{1}$ Jennifer Raftis, ${ }^{1}$ \\ Catherine McGuinnes, ${ }^{1}$ Andrew Deans, ${ }^{1}$ David E Newby, ${ }^{3}$ Nicholas L Mills, ${ }^{3}$ \\ William MacNee ${ }^{1}$
}

\section{See Editorial, p 745}

${ }^{1}$ ELEGI/Colt Laboratories, Centre for Inflammation Research, Edinburgh University, Edinburgh, UK

${ }^{2}$ Department of Pubic Health, NHS Fife, Leven, UK

${ }^{3}$ Centre for Cardiovascular Science, Edinburgh University, Edinburgh, UK

\section{Correspondence to} Dr William MacNee, ELEGI Colt Laboratory, Queen's Medical Research Institute, The University of Edinburgh, 47 Little France Crescent, Edinburgh EH16 4TJ, UK:

w.macnee@ed.ac.uk

Received 13 December 2010 Accepted 17 March 2011

Published Online First

20 April 2011

\begin{abstract}
Rationale Chronic obstructive pulmonary disease (COPD) is associated with systemic inflammation and cardiovascular disease. Interaction between inflammatory cells and activated platelets is important in the pathogenesis of atherothrombosis and may contribute to cardiovascular risk in patients with COPD. Objectives To assess platelet-monocyte aggregation in patients with COPD and matched controls, and in patients with an acute exacerbation of COPD.

Methods 18 men with COPD and 16 male controls matched for age and cigarette smoke exposure were recruited. A further 12 patients were investigated during and at least 2 weeks after hospitalisation for an acute exacerbation. Platelet-monocyte aggregation and platelet P-selectin expression were determined using flow cytometry.
\end{abstract}

Results Patients with COPD had increased circulating platelet-monocyte aggregates compared with controls (mean (SD) 25.3 (8.3)\% vs 19.5 (4.0)\%, $p=0.01$ ). Platelet-monocyte aggregation was further increased during an acute exacerbation compared with convalescence (32.0 (11.0)\% vs $25.5(6.4) \%, p=0.03)$. Platelet P-selectin expression and soluble P-selectin did not differ between groups.

Conclusions Patients with stable COPD have increased circulating platelet-monocyte aggregates compared with well-matched controls. Platelet activation is further increased in patients with COPD during an acute exacerbation. These findings identify a novel mechanism to explain the increased cardiovascular risk in COPD and suggest platelet inhibition as a plausible therapeutic target.

\section{INTRODUCTION}

Chronic obstructive pulmonary disease (COPD) is an independent risk factor for cardiovascular disease, ${ }^{1}$ although the mechanisms responsible for this association remain unclear. Systemic inflammation is recognised as an important determinant of atherosclerosis, ${ }^{2}{ }^{3}$ and COPD is characterised by both pulmonary and systemic inflammation. It has been postulated that low-grade systemic inflammation in patients with COPD may explain this increase in cardiovascular risk. ${ }^{4}$ Inflammatory pathways are upregulated further during an acute exacerbation and may plausibly precipitate an acute cardiovascular event. ${ }^{5}$

Inflammatory cells and cytokines have been implicated in atheromatous plaque formation and coronary thrombosis. ${ }^{6}$ Following vascular injury and endothelial denudation, circulating platelets

\section{Key messages}

What is the key question?

- Is COPD associated with increased platelet activation, a marker of cardiovascular risk, and is this further increased during exacerbations?

What is the bottom line?

- Platelet-monocyte aggregates are increased in stable COPD and are even higher during exacerbations of COPD.

Why read on?

- Platelet activation represents a novel mechanism by which cardiovascular risk may be increased in stable COPD and during exacerbations.

become activated, upregulating expression of cell surface receptors such as P-selectin and CD40 ligand to facilitate adhesion to the arterial wall. Activated platelets release inflammatory chemokines and recruit inflammatory cells to form platelet-monocyte aggregates, an early process in atherothrombosis. ${ }^{7}$ Circulating platelet-monocyte aggregates are considered a sensitive measure of platelet activation and are raised in patients with acute coronary syndromes, smokers and in those with rheumatoid arthritis. ${ }^{8-10}$

We hypothesise that platelet activation will be increased in patients with stable COPD and acute exacerbations, and may represent a link between inflammation and cardiovascular disease in these patients. We therefore measured markers of platelet activation, including platelet-monocyte aggregates, in patients with stable COPD and matched controls, and in patients during an acute exacerbation and in convalescence.

\section{METHODS}

We compared measures of platelet activation between patients with COPD and matched controls (study 1) and examined the effect of acute exacerbation by comparing platelet activation in patients during an exacerbation and in convalescence (study 2). These studies were approved by the Lothian regional ethics committee and conducted with the written informed consent of all participants.

Study 1: Patients with COPD and matched controls Eighteen men with COPD and 16 male controls were recruited from primary care and a hospital 
respiratory outpatient clinic at the Royal Infirmary of Edinburgh and matched for age and prior smoking habit. Ex-smokers of at least 6 months with a smoking history of $\geq 10$ pack-years were included. Control subjects had normal spirometry and no history of respiratory symptoms. Subjects with COPD had a history consistent with the disease, chronic airflow limitation on spirometry (post-bronchodilator forced expiratory volume in $1 \mathrm{~s} /$ forced vital capacity $\left(\mathrm{FEV}_{1} / \mathrm{FVC}\right.$ ) ratio $\leq 0.7$ ), stable disease (no exacerbation of COPD within the previous 6 weeks) and were not prescribed regular oral steroid therapy or long-term oxygen therapy. Exclusion criteria in both patients and controls included other respiratory disease, coronary artery disease, diabetes mellitus, hepatic and renal failure, and any systemic inflammatory condition such as rheumatoid arthritis or psoriasis, or use of medication known to affect vascular and platelet function (including statins, ACE inhibitors and clopidogrel), as used in a previous vascular study. ${ }^{11}$ Our strict inclusion and exclusion criteria were used to allow us to try to separate the effects of COPD on platelet activation from those of comorbid conditions known to influence platelet function.

Subjects were fasted overnight and blood sampled between $08.00 \mathrm{~h}$ and $10.00 \mathrm{~h}$. Subjects abstained from caffeine and alcohol for $24 \mathrm{~h}$ prior to the study and avoided all medications for at least $12 \mathrm{~h}$ prior to attendance. Height, weight and postbronchodilator spirometry were measured (Alpha Spirometer; Vitalograph, Buckingham, UK) according to American Thoracic Society/European Respiratory Society standards following venesection. ${ }^{12}$ Arterial stiffness and vascular function were measured in both patients and controls with the findings reported elsewhere. ${ }^{11}$

\section{Study 2: Acute exacerbations of COPD}

Twelve patients admitted to the Royal Infirmary of Edinburgh with an acute exacerbation of COPD were studied within $24 \mathrm{~h}$ of admission and at least 2 weeks following discharge from hospital when their condition was considered to be clinically stable. The diagnosis of acute exacerbation of COPD was made by the admitting respiratory physician. All patients had documented chronic airflow limitation on spirometry when stable (post-bronchodilator $\mathrm{FEV}_{1} / \mathrm{FVC}$ ratio $\leq 0.7, \mathrm{FEV}_{1}$ percentage predicted $<80 \%$ ) and a smoking history of $\geq 10$ pack years. Subjects with a suspected or proven alternative diagnosis for the acute deterioration in symptoms such as pneumonia, pulmonary embolism or heart failure were excluded. Patients were seen for their follow-up visit at least 2 weeks after treatment for an exacerbation with improvement in their symptoms. No patients included in study 1 were enrolled in study 2 .

\section{Blood collection}

Blood was drawn by clean venepuncture of a large antecubital vein using a 19-gauge needle with care taken to ensure a smooth blood draw. Samples were collected into tubes containing the direct thrombin inhibitor D-phenylalanine-L-prolyl-L-arginine chloromethyl (PPACK, Cambridge Biosciences, Cambridge, UK) as previously described. ${ }^{13}$ Tubes were gently inverted to ensure mixing of whole blood with anticoagulant. Further blood samples were collected for the measurement of haemoglobin, haematocrit and differential leucocyte count (Sysmex, Norderstedt, Germany), and for the measurement of blood glucose (fasting in study 1, random in study 2) and lipid profiles (Olympus Analyzers, Brea, CA, USA) in the regional clinical laboratories at the Royal Infirmary of Edinburgh. Arterial blood gases were measured at rest in study 1 (Bayer Rapidlab, Morristown, NJ, USA), as were D-dimer (bioMerieux, Basingstoke, UK) and fibrinogen levels (ACL TOP analyser, Instrumentation Laboratory, Warrington, UK). Soluble P-selectin was determined by an ELISA (R\&D Systems, Abingdon, UK) in platelet-poor plasma. Serum C-reactive protein (CRP) concentrations were measured using a highly sensitive immunonephelometric assay (Behring BN II nephelometer, Hattersheim am Main, Germany).

\section{Immunolabelling and flow cytometric analysis}

Five minutes following sample collection. whole blood was immunolabelled at room temperature for subsequent flow cytometric analysis of platelet-monocyte aggregates using monoclonal antibodies to phycoerythrin (PE)-conjugated CD14 (specifically binds to monocytes), fluoroscein isothiocynate (FITC)-conjugated CD42a (specifically binds to platelets) and isotype matched controls (Biosource, Renfrew, UK). After $20 \mathrm{~min}$ of incubation, samples were fixed and red cells lysed with FACS-Lyse solution (Becton Dickinson, Oxford, UK). Samples were analysed using a BD FACScan Flow Cytometer and data analysed using FlowJo software (Treestar, Oregon, USA). A medium flow setting was used to minimise leucocyte-platelet coincident events. Platelet-monocyte aggregates were defined as monocytes positive for CD42a (figure 1). In our laboratory the mean coefficient of variation for the percentage of plateletmonocyte aggregates is $7.8 \% .{ }^{14}$ In addition, whole blood was immunolabelled with FITC-conjugated CD42a, PE-conjugated CD62P (specifically binds to P-selectin) and isotype-matched controls (Biosource), fixed with paraformaldehyde and analysed
Figure 1 Flow cytometric analysis of platelet-monocyte aggregates in whole blood. (A) A quadrant plot of CD14-positive monocytes with isotype control was used to set the CD42a gate, adjusted for non-specific binding.

(B) The upper right quadrant shows complexes positive for CD14 (monocytes) and CD42a (platelets), platelet-monocyte aggregates.
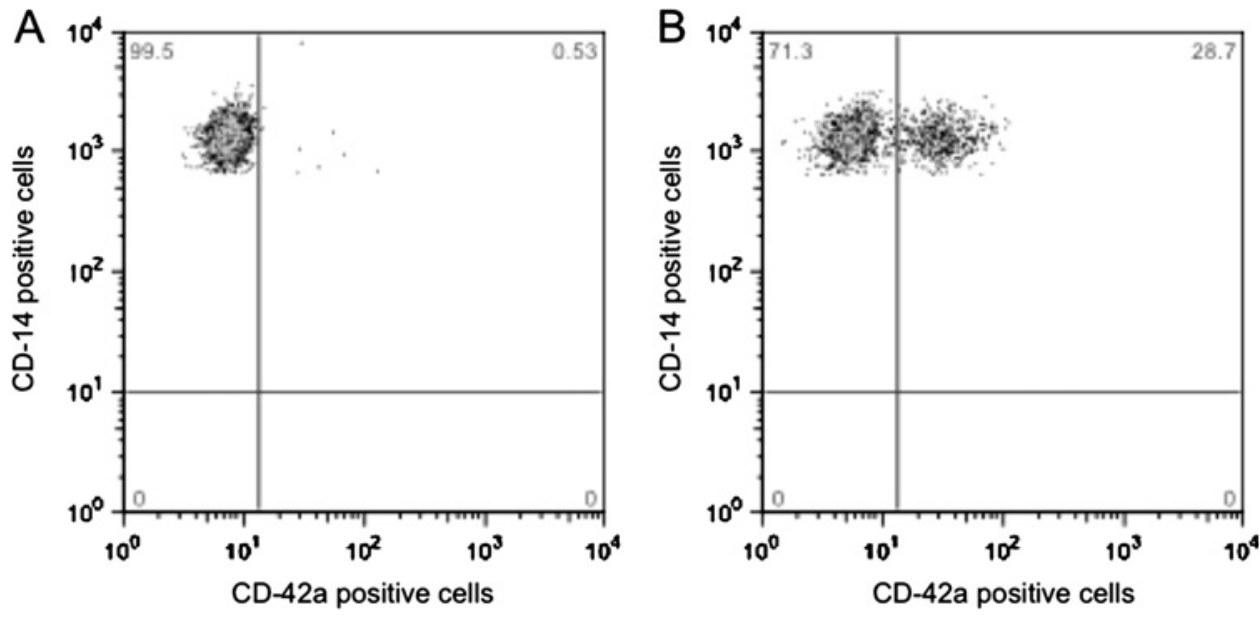
with flow cytometry to determine platelet surface expression of P-selectin.

\section{Data analysis}

Results are presented as mean (SD). Unpaired t tests were used to compare measures of platelet activation and haematological and biochemical indices between patients and controls (study 1), and paired $t$ tests were used for within-subject comparisons (study 2). CRP was log-transformed for positive skewness and the data were presented as median (IOR). There was no evidence of inhomogeneity of variance or departures from normality in any of the other data.

In exploratory analyses, associations between plateletmonocyte aggregates, age, markers of inflammation (blood neutrophils, blood leucocytes and highly sensitive CRP) and markers of disease severity (post-bronchodilator $\mathrm{FEV}_{1}$ and arterial oxygen tension) were determined using Pearson correlations. Statistical significance was taken at $\mathrm{p}<0.05$.

\section{RESULTS \\ Study 1}

Patients with COPD and controls were well matched for age and smoking history with a median pack-year history of 36 and 35, respectively (table 1). Patients with COPD had moderate to severe airflow limitation (Global Initiative in Obstructive Lung Disease (GOLD) stages 2-4) with a mean $\mathrm{FEV}_{1}$ of 1.51 and $\mathrm{FEV}_{1} / \mathrm{FVC}$ ratio of 0.42 (table 1 ).

Platelet-monocyte aggregates were increased in patients compared with matched controls (mean (SD) 25.3 (8.3)\% vs $19.5(4.0) \%, p=0.01$; figure $2 \mathrm{~A}$, table 2 ). Platelet expression of $\mathrm{P}$-selectin was higher in patients with COPD than controls, but the difference was not statistically significant (1.6 (1.2)\% vs 1.1 $(0.8) \%, p=0.16)$. Similarly, there was no difference in plasma soluble P-selectin concentrations between patients and matched controls.

Table 1 Baseline characteristics of patients with chronic obstructive pulmonary disease (COPD) and matched controls

\begin{tabular}{|c|c|c|c|}
\hline & \multicolumn{2}{|l|}{ Study 1} & \multirow{2}{*}{$\begin{array}{l}\text { Study } 2 \\
\text { COPD }\end{array}$} \\
\hline & Controls & COPD & \\
\hline $\mathrm{N}$ & 16 & 18 & 12 \\
\hline Age, years & $63(6)$ & $65(5)$ & $68(12)$ \\
\hline Male, n (\%) & $16(100)$ & $18(100)$ & $5(42)$ \\
\hline Body mass index, $\mathrm{kg} / \mathrm{m}^{2}$ & $28(4)$ & $26(4)$ & $24(6)$ \\
\hline Smoking history, pack-years* & $35(26-48)$ & $36(35-51)$ & $45(39-68)$ \\
\hline Current smoking & 0 & 0 & $3(25)$ \\
\hline \multicolumn{4}{|l|}{ Medications when stable } \\
\hline Short-acting $\beta$ agonist & 0 & $17(94 \%)$ & $8(67 \%)$ \\
\hline Long-acting $\beta$ agonist (LABA) & 0 & $3(17 \%)$ & $7(58 \%)$ \\
\hline Inhaled corticosteroids (ICS) & 0 & $1(6 \%)$ & $8(67 \%)$ \\
\hline Oral corticosteroids & 0 & 0 & $2(17 \%)$ \\
\hline Combined LABA/ICS & 0 & $13(72 \%)$ & $7(58 \%)$ \\
\hline Anticholinergics & 0 & $11(61 \%)$ & $7(25 \%)$ \\
\hline Oxygen therapy & 0 & 0 & $1(8 \%)$ \\
\hline Aspirin & $1(6 \%)$ & $2(11 \%)$ & $1(8 \%)$ \\
\hline \multicolumn{4}{|c|}{ Post-bronchodilator pulmonary function } \\
\hline $\mathrm{FEV}_{1}, \mathrm{I}$ & $3.4(0.5)$ & $1.5(0.7)$ & $0.81(0.3)$ \\
\hline FVC, I & $4.2(0.6)$ & $3.5(0.6)$ & $2.0(0.7)$ \\
\hline $\mathrm{FEV}_{1} \%$ predicted & $102(10)$ & $48(20)$ & $39(17)$ \\
\hline FVC\% predicted & $100(11)$ & $85(15)$ & $90(22)$ \\
\hline $\mathrm{FEV}_{1} / \mathrm{FVC}$ ratio & $0.79(0.05)$ & $0.42(0.13)$ & $0.42(0.12)$ \\
\hline
\end{tabular}

Values are mean (SD) or $\mathrm{n}(\%)$ unless indicated otherwise.

*Median (IQR).

$\mathrm{FEV}_{1}$, forced expiratory volume in $1 \mathrm{~s}$; FVC, forced vital capacity.
Platelet-monocyte aggregates were compared with markers of inflammation and disease severity across all subjects in study 1 . Platelet-monocyte aggregates correlated with total blood leucocyte count $(\mathrm{r}=0.45, \mathrm{p}=0.007)$ and neutrophil count $(\mathrm{r}=0.36$, $\mathrm{p}=0.03)$. There was a weak association with $\mathrm{FEV}_{1}(\mathrm{r}=0.31$, $p=0.07)$. There were no significant associations between platelet-monocyte aggregates and age, arterial oxygen or serum CRP concentration.

\section{Study 2}

Patients with an acute exacerbation had a mean age of 68 years, with lung function measured when stable similar to patients in study 1 (table 1). However, other indicators of disease severity such as the long-term use of nebuliser therapy, oxygen therapy and oral corticosteroid were more prevalent in this group. During acute exacerbations, all subjects received controlled oxygen, nebulised bronchodilators, oral corticosteroids and prophylactic low molecular weight heparin (enoxaparin $40 \mathrm{mg}$ ) for a median of 6 days following their first symptom. Clinical parameters were consistent with an acute exacerbation and had returned to normal values by the follow-up visit.

Platelet-monocyte aggregates were increased during the acute exacerbation compared with follow-up (mean (SD) $32.0(11.0) \%$ vs 25.5 (6.4)\%, $\mathrm{p}=0.03$; figure $2 \mathrm{~B}$, table 2 ). Platelet expression of P-selectin was higher during the acute exacerbation than during follow-up but the difference was not statistically significant (1.4 (2.1)\% vs $0.8(1.2) \%, \mathrm{p}=0.40)$, while there was no difference in soluble P-selectin concentrations.

\section{DISCUSSION}

Using a highly sensitive marker of platelet function, we have shown that platelet activation is increased in patients with stable COPD compared with controls matched for age and previous cigarette smoke exposure. Moreover, platelet activation is further increased in patients with COPD during an acute exacerbation. Taken together, these findings suggest that platelet function may be modified as a consequence of COPD.

We suggest that platelet activation represents a novel mechanism linking COPD, inflammation and cardiovascular disease. Platelet activation is known to predict an adverse outcome in patients with stable coronary disease ${ }^{15}$ and to identify patients likely to have recurrent cardiovascular events following percutaneous coronary intervention. ${ }^{16}$ The interaction between platelets and inflammatory cells stimulates release of chemokines and further recruitment of immune mediators which are central to the development of atherosclerotic plaque.

Platelet activation has also been implicated in structural remodelling of the pulmonary vasculature, and is thought to play a role in the pathogenesis of all forms of pulmonary arterial hypertension. ${ }^{17}$ Our findings are therefore potentially of relevance to the pulmonary vascular-as well as systemic vascular-features of COPD. We have previously reported abnormal systemic vascular function in COPD. More specifically, patients with COPD have increased arterial stiffness in comparison with controls. ${ }^{11}$ In this study we have looked at a distinct but equally important aspect of atherothrombosis and cardiovascular risk - namely, platelet activation.

Previous studies have suggested that COPD is associated with a prothrombotic and hypercoagulable state. ${ }^{18} 19$ Few studies have measured platelet activation and none has employed a direct measure of platelet function. Soluble P-selectin was increased in patients with $\mathrm{COPD}^{20}$ and inversely related to $\mathrm{FEV}_{1}{ }^{21}{ }^{21}$ However, soluble P-selectin is not a direct measure of platelet activation and may reflect P-selectin release from the 
Figure 2 (A) Patients with chronic obstructive pulmonary disease (COPD) have increased platelet-monocyte aggregates in comparison with matched controls $(p=0.01)$. (B) Patients with an exacerbation of COPD have increased platelet-monocyte aggregation compared with stable disease $(p=0.03)$. Lines represent the median values of the groups.
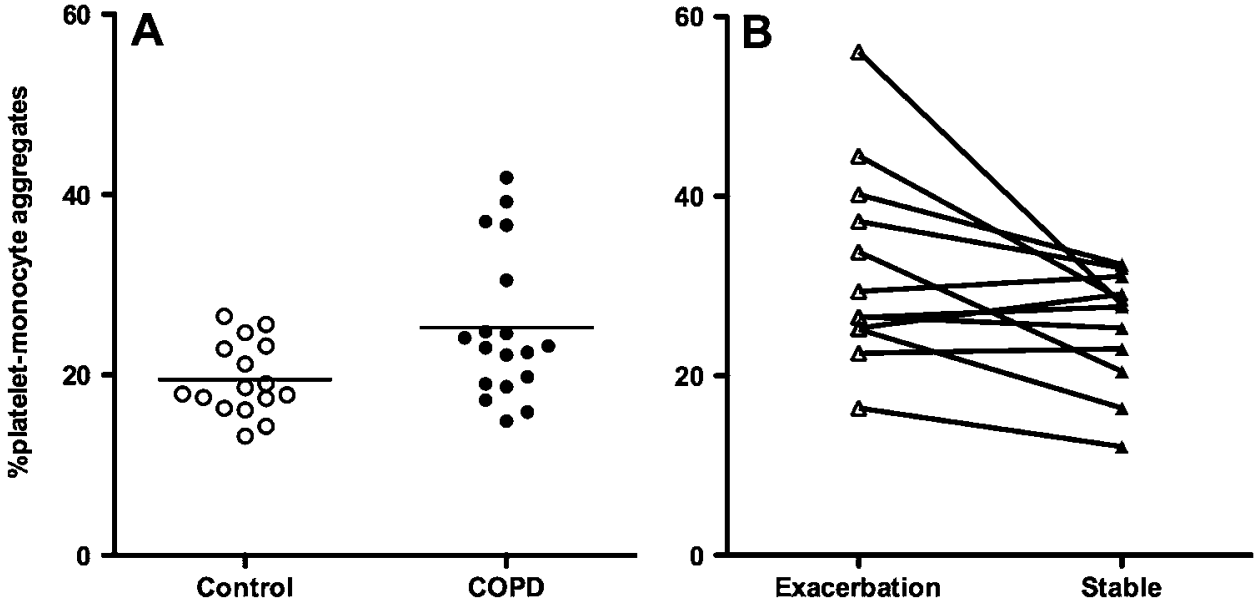

endothelium and platelet surface. ${ }^{22}$ Additionally, concentrations of soluble P-selectin may not reflect platelet surface P-selectin expression. ${ }^{23} 24$ Our study adds to the literature by demonstrating increased platelet activation using whole blood flow cytometry, which is both a sensitive and specific technique, in patients with COPD and controls well-matched for both age and, importantly, for smoking history. Furthermore, our findings are consistent across two separate cohorts, with levels of platelet-monocyte aggregation identical in patients with stable COPD from both studies.

The effects of cigarette smoking on markers of platelet activation and platelet-monocyte aggregates are well established. ${ }^{9}$ In our case-control study, all patients and controls were ex-smokers with normal exhaled carbon monoxide levels and were matched for smoking history, yet we found that plateletmonocyte aggregates were increased in patients with COPD by approximately one-third compared with controls. The magnitude of this difference was comparable to differences previously reported between smokers and non-smokers. ${ }^{9}$ This implies that platelet function may be modified as a direct consequence of
COPD, and that this potentially prothrombotic manifestation may be as important as cigarette smoking in determining cardiovascular risk in these patients. Our results are consistent with the differences seen in other inflammatory conditions associated with increased cardiovascular risk, such as rheumatoid arthritis, where platelet-monocyte aggregate levels were around $20 \%$ higher than matched controls. ${ }^{10}$ Higher plateletmonocyte aggregation is found in acute coronary syndromes, with a $30-50 \%$ increase in platelet-monocyte aggregation in comparison with patients with non-cardiac chest pain, but these levels were found during acute arterial thrombotic events. ${ }^{8} 25$

We have not identified the precise mechanism of platelet activation in patients with COPD, but a number of variablessuch as increased systemic inflammation, hypoxaemia and haemodynamic stress - that differed between patients and controls or were enhanced during acute exacerbation may be implicated.

Platelet activation is inextricably linked to local vascular inflammation, with activated platelets causing release of chemokines together with upregulation of cell surface adhesion

Table 2 Haematological and biochemical indices in patients with chronic obstructive pulmonary disease (COPD) and controls

\begin{tabular}{|c|c|c|c|c|c|c|}
\hline & \multicolumn{3}{|l|}{ Study 1} & \multicolumn{3}{|l|}{ Study 2} \\
\hline & Controls & COPD & p Value & Stable & Exacerbation & p Value \\
\hline \multicolumn{7}{|l|}{ Measures of platelet activation } \\
\hline Platelet-monocyte aggregates, $\%$ & $19.5(4.0)$ & $25.3(8.3)$ & 0.01 & $25.5(6.4)$ & $32.0(11.0)$ & 0.03 \\
\hline P-selectin expression, \% & $1.1(0.8)$ & $1.6(1.2)$ & 0.16 & $0.8(1.2)$ & $1.4(2.1)$ & 0.40 \\
\hline Soluble P-selectin, $\mathrm{ng} / \mathrm{ml}$ & $53.0(22.2)$ & $56.5(20.3)$ & 0.65 & $53.5(17.8)$ & $61.5(20.8)$ & 0.56 \\
\hline \multicolumn{7}{|l|}{ Haematological indices } \\
\hline Leucocytes, cells, $\times 10^{9} / \mathrm{l}$ & $5.3(1.3)$ & $7.0(1.4)$ & 0.23 & $7.9(1.6)$ & $14.8(3.2)$ & $<0.001$ \\
\hline Monocytes, $\times 10^{9} / \mathrm{l}$ & $0.5(0.2)$ & $0.6(0.2)$ & 0.001 & $0.7(0.3)$ & $1.0(0.3)$ & 0.001 \\
\hline Haemoglobin, $g / l$ & $139(11)$ & $141(13)$ & 0.03 & $136(9)$ & $138(12)$ & 0.40 \\
\hline Haematocrit & $0.4(0.03)$ & $0.4(0.03)$ & 0.004 & $0.4(0.02)$ & $0.4(0.04)$ & 0.41 \\
\hline Platelets, $\times 10^{9} / 1$ & $196(31)$ & $262(68)$ & 0.001 & $290(54)$ & 333 (127) & 0.09 \\
\hline D-dimer, ng/ml & 341 (202) & $373(137)$ & 0.63 & & & \\
\hline Fibrinogen, mg/dl & $2.7(0.5)$ & $2.8(0.5)$ & 0.49 & & & \\
\hline \multicolumn{7}{|l|}{ Biochemistry } \\
\hline Total cholesterol, $\mathrm{mmol} / \mathrm{l}$ & $4.9(0.9)$ & $5.5(0.7)$ & 0.03 & $5.4(1.4)$ & & \\
\hline Glucose, $\mathrm{mmol} / \mathrm{l}$ & $5.2(0.6)$ & $5.1(0.6)$ & 0.71 & $5.5(1.7)$ & & \\
\hline C-reactive protein, $\mathrm{mg} / \mathrm{l}^{*}$ & $1.0(0.5-3.2)$ & $2.1(1.3-5.4)$ & 0.03 & $2.8(1.6-8.2)$ & $41(4-103)$ & 0.01 \\
\hline Arterial oxygenation, $\mathrm{kPa}$ & $12.3(1.6)$ & $10.5(1.7)$ & 0.004 & & & \\
\hline
\end{tabular}

Values are mean (SD) unless indicated otherwise.

$\mathrm{p}$ Values calculated using independent samples $t$ test in study 1 and paired t tests in study 2. C-reactive protein was log-transformed for analysis.

*Median (IQR) 
molecules that drive monocyte recruitment, platelet-monocyte interaction and adherence to denuded endothelium. ${ }^{26}$ Furthermore, leucocytes recruited by chemotaxis cause activation of platelets. ${ }^{27}$ We identified increases in the number of peripheral blood leucocytes and neutrophils as well as higher serum concentrations of CRP in patients with COPD compared with control subjects. Circulating leucocyte and neutrophil concentrations were associated with platelet-monocyte aggregates, supporting the hypothesis that systemic inflammation in COPD may contribute to platelet activation in this condition. This mechanism is thought to be important in other chronic inflammatory conditions with increased platelet-monocyte aggregates such as rheumatoid arthritis and type 1 diabetes mellitus. ${ }^{10}{ }^{28}$ Although there was not a significant relationship between CRP and platelet-monocyte aggregates, there was a positive association and this may have been revealed in a study of larger numbers.

Alternative mechanisms through which platelet activation may occur in patients with COPD include hypoxia, tachycardia and hyperglycaemia. ${ }^{29} 30$ These factors may cause further platelet activation during an acute exacerbation and explain the association between lower respiratory tract infection and acute myocardial infarction. ${ }^{31}$ Further studies using cellular and animal models are needed to elucidate the relative importance of these mechanisms.

Interestingly, patients with COPD had higher platelet counts than controls although levels remained within the normal range. An increase in platelet count per se has been associated with adverse cardiovascular outcomes in both healthy persons and patients with acute myocardial infarction. ${ }^{32} 33$ Previous studies have suggested that anaemia is associated with an increased morbidity and mortality in patients with COPD independent of disease severity, ${ }^{34}{ }^{35}$ but the relationship between platelet count and clinical outcome has not been examined. Thus, in comparison with controls, patients with COPD not only have greater platelet activation but also increased numbers of platelets, and both may increase cardiovascular risk.

Platelet counts and platelet-monocyte aggregates may be more than simply markers of cardiovascular risk, with platelet activation a potential target for therapy. Platelet-monocyte aggregates form independently of the cyclo-oxygenase pathway and thus are not modified by aspirin therapy. ${ }^{36}$ Population studies and controlled trials are needed to determine whether aspirin is an effective antiplatelet therapy in COPD and whether the regular use of antiplatelet agents could prevent cardiovascular events in patients with COPD.

\section{Limitations of the study}

Although selection bias is possible in all case-control studies, the groups in study 1 were well-matched for age, smoking and other clinical characteristics and, as such, we do not think this can explain the reported differences in platelet activation. We did not match on weight or body mass index as we were interested in the systemic effects of COPD and, since reduced weight is a systemic effect of COPD, we did not want to over-match patients and controls. In study 2 we examined platelet activation within $24 \mathrm{~h}$ of admission with an exacerbation of COPD and at least 2 weeks post-exacerbation, while there is some evidence that the effects of acute exacerbations may persist beyond 90 days. $^{37}$ However, this would probably cause an underestimation of an effect of exacerbation on platelet activation. Additionally, we were unable to impose the same restrictions on patients with acute exacerbations as we could in the stable condition, so differences in medication, dietary intake or other environmental factors may have contributed to the platelet-monocyte aggregation observed during exacerbations. However, imposing such restrictions is impractical in these patients, and medications used during acute exacerbationssuch as steroids and prophylactic low molecular weight heparin-do not influence platelet activation. ${ }^{38} 39$

\section{CONCLUSION}

Using a highly sensitive marker of platelet activation, we have shown that platelet-monocyte aggregates are increased in patients with stable COPD independent of cigarette smoke exposure. Platelet activation was further increased in patients during an acute exacerbation. Our findings suggest that platelet function may be modified as a direct consequence of COPD, and identify platelet activation as an important prothrombotic manifestation of the disease which may be a useful therapeutic target in COPD.

Funding This research was funded primarily by a Chief Scientist Office Project Grant (CZB/4/424). DAMc is supported by a Chest, Heart and Stroke Scotland Research Fellowship (R40329). NLM is supported by a British Heart Foundation Intermediate Clinical Research Fellowship (FS/10/024). JR is supported by a Department of Health Policy Research Program Grant (PR-NT-0208-10025).

\section{Competing interests None}

Ethics approval This study was conducted with the approval of the Lothian regional ethics committee.

Contributors JDM and DAMc contributed equally to this manuscript.

Provenance and peer review Not commissioned; externally peer reviewed.

\section{REFERENCES}

1. Curkendall SM, DeLuise C, Jones JK, et al. Cardiovascular disease in patients with chronic obstructive pulmonary disease, Saskatchewan Canada cardiovascular disease in COPD patients. Ann Epidemiol 2006;16:63-70.

2. Ridker PM, Hennekens $\mathrm{CH}$, Buring JE, et al. C-reactive protein and other markers of inflammation in the prediction of cardiovascular disease in women. $N$ Engl J Med 2000;342:836-43.

3. Ridker PM, Cushman M, Stampfer MJ, et al. Inflammation, aspirin, and the risk of cardiovascular disease in apparently healthy men. $N$ Engl J Med 1997:336:973-9.

4. MacNee W, Maclay J, Mcallister D. Cardiovascular injury and repair in chronic obstructive pulmonary disease. Proc Am Thorac Soc 2008;5:824-33.

5. McAllister D, Maclay J, Cheuk S, et al. Acute Coronary Events During Acute Exacerbations of Chronic Obstructive Pulmonary Disease [abstract]. European Respiratory Society Congress programme 2007:718s:4250.

6. Libby $\mathbf{P}$, Ridker PM, Maseri A. Inflammation and atherosclerosis. Circulation 2002;105:1135-43.

7. Davì G, Patrono C. Platelet activation and atherothrombosis. N Eng/ J Med 2007;357:2482-94

8. Sarma J, Laan CA, Alam S, et al. Increased platelet binding to circulating monocytes in acute coronary syndromes. Circulation 2002;105:2166-71.

9. Harding S, Sarma J, Josephs D, et al. Upregulation of the CD40/CD40 ligand dyad and platelet-monocyte aggregation in cigarette smokers. Circulation 2004;109:1926-9.

10. Joseph JE, Harrison P, Mackie IJ, et al. Increased circulating platelet-leucocyte complexes and platelet activation in patients with antiphospholipid syndrome, systemic lupus erythematosus and rheumatoid arthritis. Br J Haematol 2001;115:451-9.

11. Maclay JD, McAllister DA, Mills NL, et al. Vascular dysfunction in chronic obstructive pulmonary disease. Am J Respir Crit Care Med 2009;180:513-20.

12. Miller MR, Hankinson J, Brusasco V, et al. Standardisation of spirometry. Eur Respir J 2005:26:319-38.

13. Harding SA, Din JN, Sarma J, et al. Flow cytometric analysis of circulating platelet-monocyte aggregates in whole blood: methodological considerations Thromb Haemost 2007:98:451-6.

14. Din J, Harding S, Valerio C, et al. Dietary intervention with oil rich fish reduces platelet-monocyte aggregation in man. Atherosclerosis 2008;197:290-6.

15. Christie DJ, Kottke-Marchant K, Gorman RT. Hypersensitivity of platelets to adenosine diphosphate in patients with stable cardiovascular disease predicts major adverse events despite antiplatelet therapy. Platelets 2008;19:104-10.

16. Gianetti J, Parri MS, Sbrana S, et al. Platelet activation predicts recurrent ischemic events after percutaneous coronary angioplasty: a 6 months prospective study. Thromb Res 2006;118:487-93.

17. Humbert M, Morrell N, Archer S, et al. Cellular and molecular pathobiology of pulmonary arterial hypertension. J Am Coll Cardiol 2004;43:13-24. 
18. Ashitani J, Mukae H, Arimura Y, et al. Elevated plasma procoagulant and fibrinolytic markers in patients with chronic obstructive pulmonary disease. Intern Med 2002; 41:181-5

19. Davi G, Basili S, Vieri M, et al. Enhanced thromboxane biosynthesis in patients with chronic obstructive pulmonary disease. The Chronic Obstructive Bronchitis and Haemostasis Study Group. Am J Respir Crit Care Med 1997:156:1794-9.

20. Ferroni P, Basili S, Martini F, et al. Soluble P-selectin as a marker of platelet hyperactivity in patients with chronic obstructive pulmonary disease. J Investig Med 2000;48:21-7.

21. Walter RE, Wilk JB, Larson MG, et al. Systemic inflammation and COPD: the Framingham Heart Study. Chest 2008;133:19-25.

22. Fijnheer R, Frijns CJ, Korteweg J, et al. The origin of P-selectin as a circulating plasma protein. Thromb Haemost 1997;77:1081-5.

23. Gurbel PA, Kereiakes DJ, Serebruany VL. Soluble P-selectin is not a surrogate marker for platelet P-selectin: evidence from a multicenter chest pain study group. J Thromb Thrombolysis 2000;10:15-22.

24. Kamath S, Blann AD, Caine GJ, et al. Platelet P-selectin levels in relation to plasma soluble P-selectin and beta-thromboglobulin levels in atrial fibrillation. Stroke 2002;33:1237-42

25. Michelson AD, Barnard MR, Krueger LA, et al. Circulating monocyte-platelet aggregates are a more sensitive marker of in vivo platelet activation than platelet surface P-selectin: studies in baboons, human coronary intervention, and human acute myocardial infarction. Circulation 2001;104:1533-7.

26. Gawaz $\mathbf{M}$, Langer $\mathrm{H}$, May AE. Platelets in inflammation and atherogenesis. J Clin Invest 2005;115:3378-84.

27. Del Maschio A, Evangelista V, Rajtar G, et al. Platelet activation by polymorphonuclear leukocytes exposed to chemotactic agents. Am J Physiol 1990;258:H870-9.

28. Harding SA, Sommerfield AJ, Sarma J, et al. Increased CD40 ligand and platelet-monocyte aggregates in patients with type 1 diabetes mellitus. Atherosclerosis 2004;176:321-5.
29. Diodati JG, Cannon RO 3rd, Epstein SE, et al. Platelet hyperaggregability across the coronary bed in response to rapid atrial pacing in patients with stable coronary artery disease. Circulation 1992;86:1186-93.

30. Vaidyula VR, Boden G, Rao AK. Platelet and monocyte activation by hyperglycemia and hyperinsulinemia in healthy subjects. Platelets 2006;17:577-85.

31. Smeeth L, Thomas SL, Hall AJ, et al. Risk of myocardial infarction and stroke after acute infection or vaccination. N Engl J Med 2004;351:2611-18.

32. Thaulow E, Erikssen J, Sandvik L, et al. Blood platelet count and function are related to total and cardiovascular death in apparently healthy men. Circulation 1991;84:613-17.

33. Ly HQ, Kirtane AJ, Murphy SA, et al. Association of platelet counts on presentation and clinical outcomes in ST-elevation myocardial infarction (from the TIMI Trials). Am J Cardiol 2006;98:1-5.

34. Cote C, Zilberberg MD, Mody SH, et al. Haemoglobin level and its clinical impact in a cohort of patients with COPD. Eur Respir J 2007:29:923-9.

35. Similowski T, Agustí A, Macnee $W$, et al. The potential impact of anaemia of chronic disease in COPD. Eur Respir J 2006:27:390-6.

36. Klinkhardt U, Bauersachs R, Adams J, et al. Clopidogrel but not aspirin reduces $\mathrm{P}$-selectin expression and formation of platelet-leukocyte aggregates in patients with atherosclerotic vascular disease. Clin Pharmacol Ther 2003; 73:232-41.

37. Seemungal TA, Donaldson GC, Bhowmik A, et al. Time course and recovery of exacerbations in patients with chronic obstructive pulmonary disease. Am J Respir Crit Care Med 2000;161:1608-13.

38. Schuerholz T, Keil 0, Wagner T, et al. Hydrocortisone does not affect major platele receptors in inflammation in vitro. Steroids 2007:72:609-13.

39. Harding SA, Din JN, Sarma J, et al. Promotion of proinflammatory interactions between platelets and monocytes by unfractionated heparin. Heart 2006:92:1635-8.

\section{Journal club}

\section{SOX9 upregulation in lung adenocarcinoma}

Several SOX genes are involved in lung development and may have functional roles within the lung. SOX9 (a transcription factor) has been shown to be involved in several types of cancer. In this lab-based study, SOX9 expression was investigated in 29 lung adenocarcinoma and 28 normal lung samples using silico data mining, quantitative reverse transcription-PCR and immunohistochemistry.

SOX9 was upregulated at both the mRNA and protein level in the majority of lung adenocarcinomas compared with normal tissue. In addition, recurrent lung adenocarcinoma tissue showed significantly higher SOX9 expression than that of a primary lesion. RNA interference technique and microarray gene expression further demonstrated that knockdown of SOX9 inhibited cell growth, downregulated CDK4 and upregulated p21. These are all important cell cycle regulators and their expression could mediate SOX9-related alteration in cancer cell growth.

SOX9 may be an important biomarker in lung adenocarcinoma which could aid in the early detection and diagnosis of NSCLC. This study builds on our existing knowledge of the role of the molecules involved in the pathogenesis of lung adenocarcinoma.

- Jiang SS, Fang WT, Hou YH, et al. Upregulation of SOX9 in lung adenocarcinoma and its involvement in the regulation of cell growth and tumorigenicity. Clin Cancer Res 2010;16:4363-73.

\section{McKinlay}

Correspondence to L McKinlay, Clinical Research Fellow, University of Dundee, Level 7 Cardiovascular and Lung Biology, Ninewells Hospital, Dundee DD19SY, UK; I.mckinlay@dundee.ac.uk

Published Online First 3 November 2010

Thorax 2011;66:774. doi:10.1136/thx.2010.153536 